\title{
The usefulness of microscopic observation for drug susceptibility of Mycobacterium tuberculosis complex in routine clinical microbiology laboratory
}

\author{
S.E. MSHANA ${ }^{1,2^{*}}$, C. IMIRZALIOGLU ${ }^{2}$, E. DOMANN ${ }^{2}$ and T. CHAKRABORTY ${ }^{2}$ \\ ${ }^{1}$ Weil Bugando University College of Health Sciences, P.O. Box 1464, Mwanza, Tanzania \\ ${ }^{2}$ Justus-Liebig University, Institute of Medical Microbiology, Giessen, Germany
}

\begin{abstract}
Clinical management of tuberculosis (TB) cases in developing countries is hampered by the lack of a simple and effective diagnostic test. Correct diagnosis of TB is needed to improve treatment, reduce transmission, and control development of drug resistance. This study was undertaken to establish microscopic observation for drug susceptibility (MODS) in clinical microbiology routine. Thirty Mycobacterium tuberculosis isolates and four smear positive sputum specimens were tested for susceptibility to isoniazid, rifampicin, ethambutol and streptomycin using MODS. Results were compared to gold standard methods used at a TB reference laboratory. The median turn around time (TAT) was six days for both direct and indirect assays. Results for rifampicin were $100 \%$ concordant with the reference laboratory and those of ethambutol, streptomycin and isoniazid were $97 \%, 94 \%$ and $94 \%$ concordant, respectively. In all discordant cases MODS categorize the isolates as resistant. Using SYBR Green to detect growth, there was clear increase in fluorescence for cultures of drug-resistant strains when compared to culture of sensitive strains. The discrepancy in these cases can be explained by the fact that in MODS any growth in drug-containing wells is labelled as resistance and can be resolved using SYBR Green. MODS can therefore, be considered as a reliable and fast method which could be used as routine in Clinical Microbiology Laboratory in a TB endemic area.
\end{abstract}

Keywords: tuberculosis, drug, susceptibility, Mycobacterium tuberculosis

\section{Introduction}

Tuberculosis (TB) is one of the most common infectious diseases in the world with over 8 million new cases and 2-3 million deaths annually (WHO, 2009). The treatment of tuberculosis is based on multiple drugs administered for a period of 6-12 months depending on the location of the disease. A cure rate of more than $95 \%$ is possible but the effectiveness is compromised when drugs are taken inappropriately due to poor patient compliance (Espinal et al., 2000). Morbidity and mortality are higher in drug resistant cases particularly multi-drug resistance-TB (MDR-TB) (WHO, 2004). Primary drug resistance in the world ranges from 0 $16 \%$ and acquired resistance rate ranges from 0-48\% (Dooley et al., 1992; Cohn et al., 1997; $\mathrm{WHO}, 2009)$. In most parts of the developing countries the situation of MDR-TB is not known and there is no routine drug susceptibility test in the existing clinical microbiology laboratories (WHO, 2008).

Advanced laboratories can detect TB cases within 7 to 14 days using liquid culturing systems such as BACTEC or Mycobacterium growth index tube (MGIT). The use of molecular techniques can shorten this period to hours in a certain amount of cases (Luz et al., 2000).
Most of reference TB laboratories in developing countries use solid media (Lowenstein Jensen media) for isolation and drug susceptibility assay. With this approach detection takes about 3-8 weeks and drug susceptibility testing takes additional 3 weeks (Madison et al., 2006). The delay in detection and determination of drug susceptibility results makes control of tuberculosis difficult. A patient with undetected MDR-TB will continue to attend clinic until after 3 months of deteriorating health, the illness threshold is surpassed and drug susceptibility test (DST) is requested. All this time the patient will have exposed others to MDR tuberculosis (David, 2007).

Microscopic observation drug susceptibility (MODS) was first described in 2000. The method is fast, cheap and comparable to gold standard methods (Luz et al., 2000). The MODS assay for detection of tuberculosis and multidrug-resistant tuberculosis directly from sputum relies on the following facts: Mycobacterium tuberculosis complex species grow faster in liquid media than in solid media, cord formation can be visualized microscopically and incorporation of drugs permits rapid and direct drug susceptibility testing together with detection of bacterial growth (Moore et al., 2004, 2006; Shiferaw et al., 2007). This method

* Correspondence : Dr. Stephen E. Mshana ; mshana72@yahoo.com 
was evaluated in large field studies in 2006 and different countries are currently evaluating the method for applicability in the specific countries (Moore et al, 2006; WHO, 2008; Brady et al, 2008). This study was conducted to evaluate the usefulness of MODS assay as a routine procedure in Clinical Microbiology Laboratory aiming at promoting this method for developing countries.

\section{Methods and Materials}

\section{Bacterial isolation}

The study was conducted at Clinical Microbiology Laboratory at Institute of Medical Microbiology in Giessen, Germany. Thirty preserved Mycobacterium tuberculosis isolates; recovered in 2006 and four smear positive sputum specimens from patients attending Infectious Clinic Unit at University Hospital, Giessen were tested using MODS as described previously (Luz et al., 2000; Moore et al., 2004). The results were compared with results from Borstel Germany TB Reference Laboratory, Middlebrook 7H9 broth (Becton Dickinson, Sparks, Md.) supplemented with 10\% oleic acid, albumin, dextrose (OADC) and $0.2 \%$ glycerol (Sigma) was prepared as per manufacturer instruction: $4.7 \mathrm{~g}$ of the powder was mixed (7H9 Base powder) in $900 \mathrm{ml}$ of distilled water containing $2 \mathrm{ml}$ glycerol, then was autoclaved at $121^{\circ} \mathrm{C}$ for 10 minutes. Aseptically $100 \mathrm{ml}$ of OADC was added and $7.2 \mathrm{ml}$ of $7 \mathrm{H} 9$ broth was dispensed into $15 \mathrm{ml}$ sterile tube followed by $160 \mu 1$ of PANTA antibiotic mixture (BD Franklin Lakes, NJ USA 07417) was added to make final concentration of PANTA $20 \mu \mathrm{l} / \mathrm{ml}$.

\section{Susceptibility assay}

Indirect and direct susceptibility assays were performed as recommended in previous studies (Luz et al., 2000; Shiferaw et al., 2007). Recommended critical concentrations and one higher concentration were tested for each drug. For indirect assay isolates from Mycobacterium growth index tube (MGIT) were taken and vortexed and left to settle for $20 \mathrm{~min}$. , the supernatant was diluted using $7 \mathrm{H} 9$ broth to $0.5 \mathrm{McF}$ arland equivalents. The $800 \mu$ of diluted sample was added into $7.2 \mathrm{ml}$ of 7H9 broth. For direct susceptibility assay, the pellets of decontaminated sputum were reconstituted with $1 \mathrm{ml}$ of $7 \mathrm{H} 9$ broth and $800 \mu \mathrm{l}$ of the mixture was added in $7.2 \mathrm{ml} 7 \mathrm{H} 9$ broth. Using prepared working sheet, $540 \mu \mathrm{l}$ of the mixture $(7.2 \mathrm{ml}$ of $7 \mathrm{H} 9$ and $800 \mu \mathrm{l}$ of sample) was distributed into corresponding 24 wells plates (Sigma). Two wells without antibiotics and the other two wells of different concentration of antibiotics. Stock solutions of antibiotics were made as follows: Isoniazid (INH) $1 \mathrm{mg} / \mathrm{ml}$ with distilled water, rifampicin (RIF) $1.0 \mathrm{mg} / \mathrm{ml}$ with methanol, ethambutol (ETH) $2.5 \mathrm{mg} / \mathrm{ml}$ with distilled water and $2.0 \mathrm{mg} / \mathrm{ml}$ streptomycin (STR) with distilled water. Solutions were filter sterilized using $0.22 \mu \mathrm{m}$ filters and $1 \mathrm{ml}$ aliquots were stored at $-20^{\circ} \mathrm{C}$. Working solutions were made by diluting stock solutions with sterile distilled water. Concentrations of working solutions were as follows: isoniazid 1 and $4 \mu \mathrm{g}$ $/ \mathrm{ml}$, rifampicin 10.0 and $5.0 \mu \mathrm{g} / \mathrm{ml}$, ethambutol 25 and $50 \mu \mathrm{g} / \mathrm{ml}$ and streptomycin 20 and $60 \mu \mathrm{g} /$ $\mathrm{ml}$ (David et al., 2007).

In each well, $60 \mu \mathrm{l}$ of working solution of antibiotics was added. $60 \mu \mathrm{l}$ of $7 \mathrm{H} 9$ broth was applied to the drug free wells. The final concentrations of the examined drugs were 0.1 and $0.4 \mu \mathrm{g} / \mathrm{ml} \mathrm{INH}, 0.5$ and $1 \mu \mathrm{g} / \mathrm{ml}$ RIF, 2.5 and $5 \mu \mathrm{g} / \mathrm{ml}$ ETH and 2 and $6 \mu \mathrm{g} / \mathrm{ml}$ STR. Plates were covered and sealed completely with parafilm (Chicago, IL 60631) and incubated at $37^{\circ} \mathrm{C}$ for up to 40 days. Culture plates were examined under inverted light microscope (Leitz $\mathrm{GmbH}$ ) at magnification of 32x daily from day 4 to 7 and alternate day from day 9 to 21 and twice weekly from day 26 to 40 .

Positive culture was identified by cord formation characteristic of $M$. tuberculosis (Figure 1). Drug free wells were examined first. In case of growth in these wells and absence of growth in drug contained wells, the isolate was considered susceptible. Bacterial and fungal contamination was recognized by rapid overgrowth and clouding in the wells. The first day of visible growth was noted. Plates were examined in subsequent days to confirm susceptibility results. This was done two days after growth in the drug free wells was detected. Two examiners read the plates independently. In discordant cases a third person was used to resolve the difference. The results were compared to results from reference laboratory.

The use of SYBR Green stain (Molecular Probes, USA) to quantify mycobacterial growth was also investigated. Portions $(200 \mu \mathrm{l})$ of cultures from 10 strains growing at day 0,4 and 7 in culture broth with and without antibiotics were heat-inactivated before staining with $10 \mu \mathrm{l}$ of SYBR Green and examined for fluorescence emission (Molecular Devices, USA). 


\section{Results}

The median turn around time (TAT) was 6 days (range $=4-15$ days). The susceptibility results were $100 \%$ concordant with reference laboratory for rifampicin, $97 \%$ concordant for ethambutol and $94 \%$ concordant for isoniazid and streptomycin (Table 1).
Two isolates were confirmed to be Mycobacterium tuberculosis complex using an inverse hybridization genotyping assay (Hain Lifescience $\mathrm{GmbH}$ ) and they had TAT of 6 and 7 days. One which had cord like morphology and overgrowth within $48 \mathrm{~h}$ was identified as Mycobacterium chelonae. The fourth direct sample was positive after 8 days and exhibited star-like growth. This was identified as Mycobacterium

Table 1: Susceptibility results of the isolates of Mycobacterium tuberculosis complex

\begin{tabular}{llllllc}
\hline Drug & \multicolumn{2}{c}{ MODS } & \multicolumn{2}{c}{ TB Reference Lab } & \multicolumn{2}{c}{ Agreement } \\
& Sensitive & Resistant & Sensitive & Resistant & Concordant & Discordant \\
\hline Rifampicin & $33(97 \%)$ & $1(3.0 \%)$ & $33(97 \%)$ & $1(3.0 \%)$ & $34(100 \%$ & 0 \\
Ethambutol & $31(92 \%)$ & $3(8.0 \%)$ & $32(94 \%)$ & $2(6.0 \%)$ & $33(97 \%)$ & $1(3.0 \%)$ \\
Isoniazid & $26(76 \%)$ & $8(24 \%)$ & $28(82 \%)$ & $6(18 \%)$ & $32(94 \%)$ & $2(6.0 \%)$ \\
Streptomycin & $27(79 \%)$ & $7(21 \%)$ & $29(86 \%)$ & $5(14 \%)$ & $32(94 \%)$ & $2(6.0 \%)$ \\
\hline
\end{tabular}

MODS: Microscopic Observation Drug Susceptibility

Sybr green showed clear increase in fluorescence intensity in drug resistant wells compared to drug sensitive wells (Figures 2 and 3).

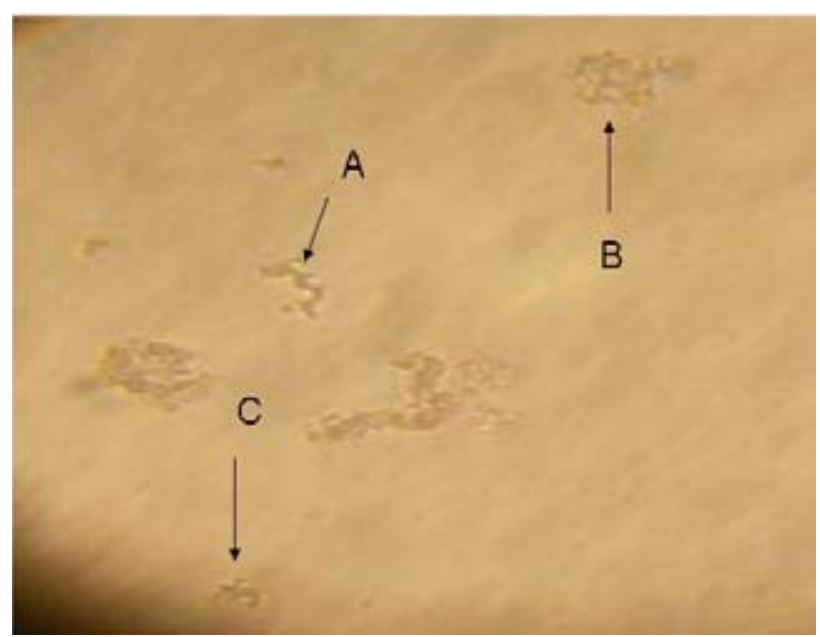

Figure 1: Characteristics serpentine structure of $M$. tuberculosis complex colonies A, B and C grown in Middlebrook 7H9 broth of MODS, seen under inverted light microscope (32 $\mathrm{x}$ magnifications)

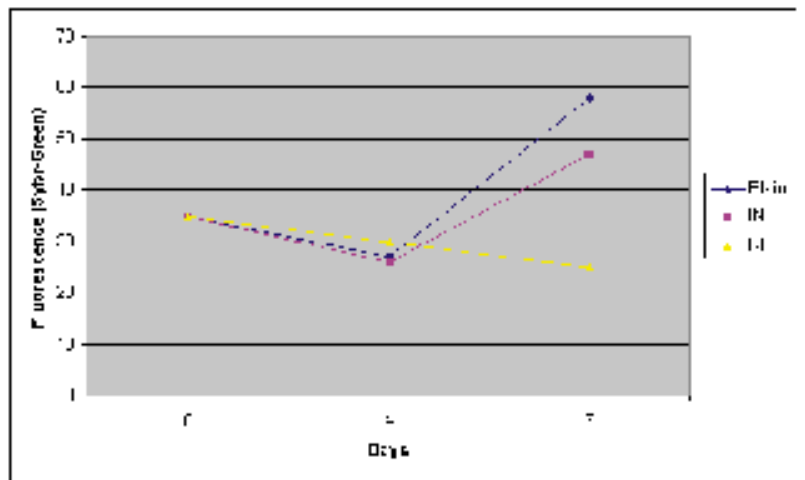

Figure 2: Sybr-Green fluorescence as an indicator for growth in an isoniazid (INH) resistant but rifampicin (RIF) sensitive $M$. tuberculosis strain celatum and it was correctly labelled resistant to isoniazid, rifampicin, ethambutol and streptomycin by MODS.

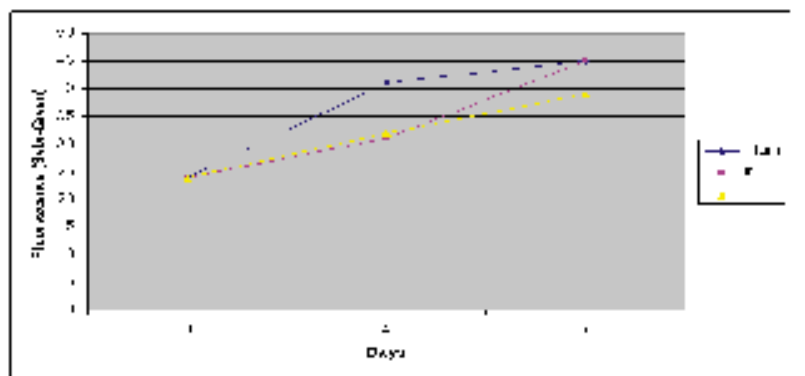

Figure 3: Sybr-Green fluorescence as an indicator for growth in an isoniazid (INH) and rifampicin (RIF) sensitive $M$. tuberculosis

\section{Discussion}

The median turn around time (TAT) observed in this study was 6 days. This is slightly lower than the TAT (7-9 days) observed by others (Luz et al., 2000; Moore et al., 2004). The TAT was comparable to MGIT, and BACTEC which have median turn around time of 14 days from receiving of specimen to susceptibility results. Using Lowenstein Jensen (LJ) slopes the median TAT for indirect assay from receiving specimens to susceptibility results was 3.5 weeks. Two weeks can be gained if LJ proportion method is used to asses drug susceptibility (Luz et al., 2000).

In four direct samples assessed, TAT was comparable to previous studies (Luz et al., 2000; Moore et al., 2004, 2006). Two members of the Mycobacterium tuberculosis complex namely, Mycobacterium chelonae and Mycobacterium celatum were identified in this study. M. celatum 
was found resistant to isoniazid, rifampicin, ethambutol and streptomycin by MODS (Moore et al., 2004). The cording growth characteristic has been found to be useful in identifying $M$. tuberculosis complex. M. chelonae and $M$. smegmatis also display cord like growth but can be easily differentiated from $M$. tuberculosis complex by markedly faster growth (Luz et al., 2000; Moore et al., 2004). M. chelonae grows fast with visible colonies within 48 h (David, 2007).

Generally in this study susceptibility results were $100 \%$ concordant to results from TB reference laboratory for rifampicin, 97\% concordant for ethambutol and 94\% concordant for isoniazid and streptomycin. In all discordant cases MODS showed resistance, rather than susceptibility. In two cases the bacteria were resistant to low concentration of streptomycin and isoniazid (Moore et al., 2004, 2006). The discordance in these cases can be explained by the fact that in MODS, as a qualitative method, any growth in drug containing wells is labelled as resistance. Due to lack of colonies, proportion could not be calculated in MODS. Decrease in antibiotic potency due to storage, inocolum variation or true resistance are other possible explanations. In the two cases of low level resistance, decrease in drug potency was suspected and retesting with new stock solution of isoniazid and streptomycin was performed. These samples were found to be sensitive with new stock solution of isoniazid and streptomycin. It was noted, that the stock solution had been stored for 10 weeks at $-20^{\circ} \mathrm{C}$ at the time of susceptibility assay. One isolate which was resistant to ethambutol on indirect assay, was also found to be resistant on direct assay to low $(25 \mu \mathrm{g} / \mathrm{ml})$ and high $(50 \mu \mathrm{g} / \mathrm{ml})$ ethambutol concentration on repeat.

The method can be used in clinical microbiology routine for both indirect susceptibility assay as in this study and for direct susceptibility assay as recommended previously (Moore et al., 2004). Using it for indirect susceptibility assay in places where indirect assay using LJ proportion method is performed as in most developing countries, it will shorten the time for susceptibility results by more than 2 weeks.

To address microscopic bias the use of SYBR Green to quantify mycobacterial growth can be used. However, further research is needed in this area. In this study clear increase in fluorescence for cultures of drug-resistant strains when compared to culture of sensitive strains was observed. Thus this modification allows quantification of growth and extends the utility of MODS as a routine laboratory tool. This method is fast and gives comparable results with standards methods. It can also be readily used as a routine method in regional and tertiary clinical microbiology laboratories in developing countries where drug-resistant tuberculosis is a major problem. Establishment of MODS in developing countries for routine M. tuberculosis susceptibility testing will help to guide TB therapy and monitor for MDR and extensively drug resistance.

Received 26 September 2008

Revised 27 March 2009

Accepted 27 March 2009

\section{References}

Brady, M.F, Coronel, J, Gilman, R.H. \& Moore, D. A. (2008) The MODS method for diagnosis of tuberculosis and multidrug resistant tuberculosis. Journal of Visualized Experiments 11 (17) p ii: 845. doi: $10.3791 / 845$.

Cohn, D.L., Bustro, F. \& Raviglinone, M.C. (1997) Drug resistant tuberculosis review of the worldwide situation and $\mathrm{WHO} /$ IUATLD Global surveillance Project. Clinical Infectious Diseases 24, 512-513.

David, A.J. (2007) Future prospects for the MODS assay in multidrug-resistant tuberculosis diagnosis. Future Microbiology 2, 97-101.

Dooley, S.W., Jarvis, W.R., Marlone, W.J. \& Synder, D.E. (1992) Multi-drug resistant tuberculosis (editorial) Annals of Internal Medicine 117, 257-258.

Espinal, M.A., Kim, S.J. \& Suarez, P.A. (2000) Standard short course chemotherapy for drug resistant tuberculosis treatment outcome in six countries. Journal of American Medical Association 283, 25372545.

Luz, C., Tien-Shun, L. \& Robert, H. (2000) Rapid and efficient detection and drug susceptibility testing of Mycobacterium tuberculosis in sputum by microscopic observation of broth cultures. Journal of Clinical Microbiology 38, 1203-1208.

Madison, B., Robinson, D. I., George W, Gross, H., Lipman, B. \& Metchok, A. (2006) Multi-center evaluation of ethambutol susceptibility testing of Mycobacterium tuberculosis by agar proportion and radiometric methods. Journal of Clinical Microbiology 36, 1214-1219.

Moore, D.A.J., Evans, C.A.W., Gilman, R.H., 
Caviedes, L., Coronel, J., Vivar, A., Sanchez, E., Pinedo, Y., Saravia, J.C., Salazar, C., Oberhelman, R., HollmDelgado, M.G., LaChira, D., Escombe, R. \& Friedland, J.S. (2006) microscopicobservation drug-susceptibility assay for the diagnosis of TB. New England Journal of Medicine 355, 539-1550.

Moore, D.A.J., Mendoza, D., Gilman, R.H., Evans, C.A.W., Hollm- Delgado,M. G., Guerra, J., Caviedes, L., Vargas, D., Ticona, E., Ortiz, J., Soto, G., Serpa, J. and the Tuberculosis Working Group in Peru (2004) Microscopic Observation Drug Susceptibility Assay, a rapid, reliable diagnostic test for multidrugresistant tuberculosis suitable for use in resource-poor settings. Journal of Clinical Microbiology 42, 32-37.

Shiferaw, G., Woldeamanuel, Y., Gebeyehu, M., Girmachew, F., Demessie, D. \& Lemma, E. (2007) Evaluation of microscopic observation drug susceptibility assay (MODS) for detection of multidrugresistant tuberculosis (MDR-TB). Journal of Clinical Microbiology doi:10.1128/ JCM.01949-06.

WHO (2004) World Health Organization/ International Union Against Tuberculosis and Lung Disease Global Project on AntiTuberculosis Drug Resistance Surveillance. Anti-tuberculosis drug resistance in the world: Report No. 3. World Health Organization Geneva, Switzerland.

WHO (2008) World Health Organization/ International Union Against Tuberculosis and Lung Disease Global Project on AntiTuberculosis Drug Resistance Surveillance. World Health Organization, Geneva, Switzerland.

WHO (2009) Global Tuberculosis: Control, Epidemiology, Strategy, Financing, World Health Organization. 